\title{
EFFicient STRATEGy-Proof EXChange AND Minimum CONSUMPTION GUARANTEES
}

\author{
by
}

Shigehiro Serizawa and John A. Weymark

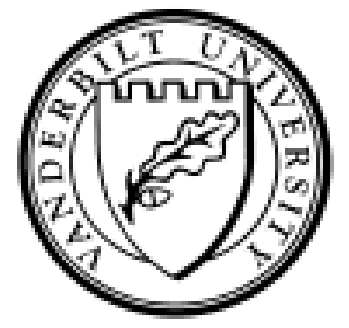

\section{Working Paper No. 02-W16R}

June 2002

Revised August 2002

\section{DEPARTMENT OF ECONOMICS \\ VANDERBILT UNIVERSITY \\ NASHVILLE, TN 37235}

www.vanderbilt.edu/econ 


\title{
Efficient Strategy-Proof Exchange and Minimum Consumption Guarantees*
}

\author{
by \\ Shigehiro Serizawa \\ Faculty of Economics, Tohoku University, \\ Kawauchi, Aoba, Sendai, Miyagi 980-8576, Japan \\ (e-mail: serizawa@econ.tohoku.ac.jp) \\ and \\ John A. Weymark \\ Department of Economics, Box 1819, Station B, \\ Vanderbilt University, Nashville, TN 37235, U.S.A. \\ (e-mail: john.weymark@vanderbilt.edu)
}

June 2002

Revised, August 2002

Running Title: Strategy-Proof Exchange

Corresponding Author: John A. Weymark

Tel: $1-615-322-1437$

Fax: 1-615-343-8493

*We have benefitted from discussions with Stephen Ching, Shinji Ohseto, Jim Schummer, and William Thomson. This article was partly written while the second author was a Visiting Professor at Université Paris 1 (PanthéonSorbonne). 


\begin{abstract}
"Efficient Strategy-Proof Exchange

and Minimum Consumption Guarantees"

by

Shigehiro Serizawa and John A. Weymark
\end{abstract}

For exchange economies with classical economic preferences, it is shown that any strategy-proof social choice function that selects Pareto optimal outcomes cannot guarantee everyone a consumption bundle bounded away from the origin. This result demonstrates that there is a fundamental conflict between efficiency and distributional goals in exchange economies if the social choice rule is required to be strategy-proof.

Journal of Economic Literature classification number: D71.

Keywords and phrases: social choice, strategy-proofness, exchange economies. 


\section{Introduction}

The main lesson to emerge from the literature that has built on the pioneering article by Hurwicz [3] on incentive compatibility in economic environments is that any strategy-proof social choice function that selects Pareto optimal (i.e., efficient) outcomes must violate some other desirable properties of a collective choice rule. For example, Satterthwaite [15, p. 44] writes that "... even though a completely general theorem has not yet been formulated and proved, it seems clear that no attractive social choice functions exist for market settings that are both strategy-proof and efficient." In this article, we provide further support for this conclusion. We show that for exchange economies with classical economic preferences, strategy-proofness and Pareto optimality jointly imply that there exists a profile of preferences in the domain of the social choice function for which someone's consumption of each good is arbitrarily close to 0 . Thus, in exchange economies, there is a fundamental conflict between efficiency and distributional goals if the social choice rule is required to be strategy-proof.

More precisely, we consider exchange economies with $n \geq 2$ individuals and $m \geq 2$ goods. The aggregate endowment of goods is fixed. A social choice function assigns a feasible allocation to each profile of preferences in its domain. It is assumed that everybody has the same set of admissible preferences.

Each individual only cares about his own consumption, so preferences are defined on the nonnegative orthant of $\mathbb{R}^{m}$. A preference is classical if it is continuous, strictly monotonic, and strictly convex, with the latter two properties only required to hold when the consumption of all goods is positive. ${ }^{1}$

A social choice function guarantees minimum consumption if there exists an $\varepsilon>0$ such that each individual is always assigned a commodity bundle at least $\varepsilon$ distance from the origin of his consumption set. Any social choice rule that violates this property is clearly unsatisfactory on distributional grounds.

Our theorem shows that a social choice function cannot guarantee minimum consumption if it is strategy-proof and chooses Pareto optimal outcomes whenever the domain of individual preferences includes the set of classical, smooth (unique supporting prices on the positive orthant), and homothetic

\footnotetext{
${ }^{1}$ The definition of a classical preference differs somewhat in some of the other articles discussed in this section. For example, some authors require strict monotonicity to hold on all of the nonnegative orthant.
} 
preferences. In particular, strategy-proofness, Pareto optimality, and minimum consumption guarantee are incompatible for the domain of all classical economic preferences.

If the endowment is privately owned and participation in the collective choice procedure is voluntary, then the social choice function is individually rational; i.e., each person is guaranteed a consumption bundle weakly preferred to his endowment. Hurwicz [3] has shown that strategy-proofness, Pareto optimality, and individual rationality are inconsistent for two-person, two-good exchange economies provided that the domain includes a sufficiently rich set of classical preferences. This impossibility theorem has recently been extended to the general $n$-person, $m$-good case by Serizawa [19]. Serizawa established his result for the same domain restriction used in our theorem. He assumed that each person has a positive endowment of some good. With the slightly stronger assumption that everyone has a positive endowment of all goods, we show that Serizawa's theorem is implied by ours. ${ }^{2}$

Our impossibility theorem is also related to the literature that investigates whether it is possible to construct a nondictatorial social choice function for an exchange economy that satisfies both strategy-proofness and Pareto optimality. With monotonic preferences, an individual is a dictator in an exchange economy if he always receives the whole endowment. For the domain of classical preferences, Zhou [21] has shown that strategy-proofness, Pareto optimality, and nondictatorship are inconsistent when there are at least two goods, but only two individuals. His theorem has been extended by Schummer [17] and Ju [5] to more restrictive domains. When there are at least three individuals, Satterthwaite and Sonnenschein [16] have shown by example how to construct efficient, strategy-proof, nondictatorial social choice functions for the domain of classical preferences. ${ }^{3}$ In their example, someone is bossy; i.e., there is an individual who can change the consumption bundle of someone else by reporting a different preference without affecting his own consumption bundle. Serizawa [18] has strengthened strategy-proofness by

\footnotetext{
${ }^{2}$ Serizawa has also shown that individual rationality can be replaced in his theorem by an equal-treatment property that requires individuals who have the same preferences for own consumption to receive commodity bundles that are indifferent to each other.

${ }^{3}$ Dasgupta, Hammond, and Maskin [2] have demonstrated that no strategy-proof, Pareto optimal, and nondictatorial social choice functions exist for $n$-person, $m$-good exchange economies when the individual preference domain is the set of all strictly monotonic and strictly convex preferences. Their result relies on the presence of discontinuous preferences in the domain.
} 
requiring that no pair of individuals can manipulate the social choice function by misreporting their preferences. He has shown that this pairwise strategyproofness condition and Pareto optimality jointly imply that the social choice function is dictatorial on the classical domain of preferences when there are two or more individuals and two or more goods.

As noted by Zhou [21], Satterthwaite and Sonnenschein's example has the property that there is some individual who always receives nothing, what he calls an inverse dictator. For the domain of classical preferences, Zhou conjectured that there must be an inverse dictator if the social choice function satisfies strategy-proofness and Pareto optimality. This is the case if there are only two people because if one person is a dictator, then the other person is an inverse dictator. Zhou's conjecture has recently been shown to be false by Kato and Ohseto [8], at least when there are four or more individuals. The social choice functions Kato and Ohseto have constructed to disprove Zhou's conjecture always assign the whole endowment to one person, but who this person is is profile-dependent. As a consequence, their social choice functions are not very appealing. ${ }^{4}$

While Zhou's conjecture is not correct, our theorems demonstrate that something similar in spirit to his conjecture is true: for an exchange economy with classical preferences, an efficient, strategy-proof social choice function must generate unacceptable distributions of the goods in some circumstances. While there need not be someone who always receives no consumption, it is nevertheless the case that at least one person must sometimes be allocated a consumption bundle arbitrarily close to the origin. ${ }^{5}$

The rest of this article is organized as follows. In Section 2, we present the model. We formally state our impossibility theorem in Section 3. We present some preliminary results that are used to help prove our theorem in Section 4. Section 5 is devoted to the proof of our theorem. Section 6 provides some concluding remarks.

\footnotetext{
${ }^{4}$ See also the related examples in Kato and Ohseto [9].

${ }^{5}$ Strategy-proofness in exchange economies has also been studied using different assumptions. For example, Barberà and Jackson [1] and Hurwicz and Walker [4] drop Pareto optimality, but adopt other restrictive assumptions. Continuous social choice functions have been analyzed by Ju [6] and Sprumont [20], among others.
} 


\section{The Model}

The set of individuals is $N=\{1, \ldots, n\}$, where $n \geq 2$. There are $m \geq 2$ private goods, denoted by $M=\{1, \ldots, m\}$. The consumption set for each individual is $\mathbb{R}_{+}^{m} \cdot{ }^{6}$ A consumption bundle for individual $i \in N$ is a vector $x^{i}=$ $\left(x_{1}^{i}, \ldots, x_{m}^{i}\right) \in \mathbb{R}_{+}^{m}$. When no specific individual is intended, the superscript is suppressed. The economy's endowment of goods is $\Omega \in \mathbb{R}_{++}^{m}$. If the resource endowment is privately owned, then $\Omega=\sum_{i \in N} \omega^{i}$, where $\omega^{i} \in \mathbb{R}_{+}^{m}$ is individual $i$ 's endowment. Endowments are fixed and publically known. An allocation is a vector $\mathbf{x}=\left(x^{1}, \ldots, x^{n}\right) \in \mathbb{R}_{+}^{m n}$. The set of feasible allocations for an $n$-person, $m$-good exchange economy with endowment $\Omega$ is

$$
X=\left\{\mathbf{x} \in \mathbb{R}_{+}^{m n} \mid \sum_{i \in N} x^{i} \leq \Omega\right\} .
$$

Let $X^{*}=X \cap \mathbb{R}_{++}^{m n}$ denote the set of feasible allocations with positive consumptions.

A preference $R$ is a complete, reflexive, and transitive binary relation on $\mathbb{R}_{+}^{m}$. The corresponding strict preference $P$ and indifference $I$ relations are defined in the usual way: for all $x, \bar{x} \in \mathbb{R}_{+}^{m}$, (i) $x P^{i} \bar{x} \leftrightarrow[x R \bar{x}$ and $\neg(\bar{x} R x)]$ and (ii) $x I \bar{x} \leftrightarrow[x R \bar{x}$ and $\bar{x} R x]$. The set of all preference orderings on $\mathbb{R}_{+}^{m}$ is $\mathcal{R}$. Each individual $i \in N$ is assumed to have a preference ordering $R^{i}$ on $\mathbb{R}_{+}^{m}$.

Given a preference $R \in \mathcal{R}$ and a consumption bundle $x \in \mathbb{R}_{+}^{m}$, the upper contour set of $R$ at $x$ is $U C(R, x)=\left\{\bar{x} \in \mathbb{R}_{+}^{m} \mid \bar{x} R x\right\}$ and the lower contour set of $R$ at $x$ is $L C(R, x)=\left\{\bar{x} \in \mathbb{R}_{+}^{m} \mid x R \bar{x}\right\}$. A preference $R \in \mathcal{R}$ is continuous if $U C(R, x)$ and $L C(R, x)$ are both closed for all $x \in \mathbb{R}_{+}^{m}$. A preference $R \in \mathcal{R}$ is strictly convex on $\mathbb{R}_{++}^{m}$ if $U C(R, x)$ is strictly convex for all $x \in \mathbb{R}_{++}^{m}$. A preference $R \in \mathcal{R}$ is strictly monotonic on $\mathbb{R}_{++}^{m}$ if for all $x, \bar{x} \in \mathbb{R}_{++}^{m}, x>\bar{x} \rightarrow x P \bar{x}{ }^{7} \quad$ A preference $R \in \mathcal{R}$ is homothetic if for all $x, \bar{x} \in \mathbb{R}_{+}^{m}$ and all $\lambda>0,(\lambda x) R(\lambda \bar{x}) \leftrightarrow x R \bar{x}$. A preference $R \in \mathcal{R}$ is smooth if for all $x \in \mathbb{R}_{++}^{m}$, there is a unique vector $p \in S^{m-1}$ such that $p$ is the normal of a supporting hyperplane to $U C(R, x)$ at $x .^{8}$

\footnotetext{
${ }^{6} \mathbb{R}_{+}^{m}$ and $\mathbb{R}_{++}^{m}$ are the nonnegative and positive orthants, respectively, of the Euclidean $m$-space $\mathbb{R}^{m}$. The origin in $\mathbb{R}^{m}$ is $0_{m}$.

${ }^{7}$ We use the following conventions for vector inequalities: for all $x, y \in \mathbb{R}^{m}$, (i) $x \geq$ $y \leftrightarrow\left[x_{k} \geq y_{k}\right.$ for all $\left.k \in M\right]$, (ii) $x>y \leftrightarrow[x \geq y$ and $x \neq y]$, and (iii) $x \gg y \leftrightarrow\left[x_{k}>y_{k}\right.$ for all $k \in M]$.

${ }^{8} S^{m-1}$ is the unit simplex in $\mathbb{R}^{m}$.
} 
A preference $R \in \mathcal{R}$ is classical if it continuous (on $\mathbb{R}_{+}^{m}$ ) and strictly convex and strictly monotonic on $\mathbb{R}_{++}^{m}$. We denote the class of classical preferences by $\mathcal{R}^{C}$. Because a classical preference is only required to be strictly convex and strictly monotonic on $\mathbb{R}_{++}^{m}$, rather than on all of $\mathbb{R}_{+}^{m}$, CobbDouglas and CES preferences are in $\mathcal{R}^{C}$. We also consider three subclasses of $\mathcal{R}^{C}$. $\mathcal{R}^{C H}$ is the class of homothetic preferences in $\mathcal{R}^{C}, \mathcal{R}^{C S}$ is the class of smooth preferences in $\mathcal{R}^{C}$, and $\mathcal{R}^{C H S}$ is the class of homothetic and smooth preferences in $\mathcal{R}^{C}$.

A preference profile is an $n$-tuple $\mathbf{R}=\left(R^{1}, \ldots, R^{n}\right) \in \mathcal{R}^{n}$. The subprofile obtained by removing $R^{i}$ from $\mathbf{R}$ is $\mathbf{R}^{-i}=\left(R^{1}, \ldots, R^{i-1}, R^{i+1}, \ldots, R^{n}\right)$. It is sometimes convenient to write the profile $\left(R^{1}, \ldots, R^{i-1}, \bar{R}^{i}, R^{i+1}, \ldots, R^{n}\right)$ as $\left(\bar{R}^{i} ; \mathbf{R}^{-i}\right)$.

Each individual is a priori restricted to having a preference in $\mathcal{D} \subseteq \mathcal{R}$. A social choice function $f: \mathcal{D}^{n} \rightarrow X$ assigns a feasible allocation to each preference profile in $\mathcal{D}^{n}$. The set $\mathcal{D}^{n}$ is the domain of the social choice function. For all $\mathbf{R} \in \mathcal{D}^{n}$, the outcome chosen can be written as $f(\mathbf{R})=$ $\left(f^{1}(\mathbf{R}), \ldots, f^{n}(\mathbf{R})\right)$, where $f^{i}(\mathbf{R})$ is the consumption bundle allocated to individual $i$ by $f$.

\section{The Impossibility Theorem}

We consider four social choice function axioms. The first of our axioms is strategy-proofness. A social choice function is strategy-proof if no one can ever obtain a preferred outcome by misreporting his preference.

Strategy-proofness. A social choice function $f$ is strategy-proof if for all $\mathbf{R} \in \mathcal{D}^{n}$, all $i \in N$, and all $\bar{R}^{i} \in \mathcal{D}, f^{i}(\mathbf{R}) R^{i} f^{i}\left(\bar{R}^{i} ; \mathbf{R}^{-i}\right)$.

A feasible allocation is (strongly) Pareto optimal if there is no other feasible allocation that would benefit someone without worsening the situation of anyone else. Formally, an allocation $\mathbf{x} \in X$ is Pareto optimal for the preference profile $\mathbf{R} \in \mathcal{D}^{n}$ if for all $\overline{\mathbf{x}} \in X,\left[\bar{x}^{i} P^{i} x^{i}\right.$ for some $\left.i \in N\right] \rightarrow\left[x^{j} P^{j} \bar{x}^{j}\right.$ for some $j \in N]$. Given $\Omega \in \mathbb{R}_{+}^{m}$, let $\mathcal{P}(\mathbf{R}, \Omega)$ denote the set of Pareto optimal allocations for the profile $\mathbf{R} \in \mathcal{D}^{n}$. A social choice function is Pareto optimal if it always choose Pareto optimal outcomes.

Pareto optimality. A social choice function $f$ is Pareto optimal if for all $\mathbf{R} \in \mathcal{D}^{n}, f(\mathbf{R})$ is Pareto optimal for $\mathbf{R}$. 
A social choice function guarantees minimum consumption if for every profile in the domain, each individual's consumption bundle is bounded away from the origin.

Minimum consumption guarantee. A social choice function $f$ gurantees minimum consumption if there exists an $\varepsilon>0$ such that for all $\mathbf{R} \in \mathcal{D}^{n}$ and all $i \in N,\left\|f^{i}(\mathbf{R})\right\| \geq \varepsilon$.

If this axiom is satisfied, everyone is ensured of receiving a consumption bundle at least distance $\varepsilon$ from the origin. Note that the value of $\varepsilon$ is profile independent. Minimum consumption guarantee is a very weak distributional requirement because $\varepsilon$ can be arbitrarily close to 0 . If this axiom is violated, there must exist a profile for which someone receives a commodity bundle arbitrarily close to the origin. ${ }^{9}$

Our final axiom only applies if endowments are privately owned. Individual rationality requires that nobody is ever made worse off than he would be consuming his endowment. When this condition is satisfied, individuals will voluntarily participate in the social choice procedure.

Individual rationality. A social choice function $f$ is individually rational if for all $\mathbf{R} \in \mathcal{D}^{n}$ and all $i \in N, f^{i}(\mathbf{R}) R^{i} \omega^{i}$.

Our impossibility theorem shows that strategy-proofness and Pareto optimality are incompatible with the minimum consumption guarantee axiom when the domain of the social choice function contains the set of profiles of classical, homothetic, and smooth preferences. In particular, these three axioms are inconsistent on the domain of classical preference profiles.

Theorem. Suppose that $\mathcal{R}^{C H S} \subseteq \mathcal{D} \subseteq \mathcal{R}$. If a social choice function $f: \mathcal{D}^{n} \rightarrow X$ satisfies strategy-proofness and Pareto optimality, then it violates minimum consumption guarantee.

The proof of this theorem may be found in Section 5. The proof involves first showing that the axioms are inconsistent when $\mathcal{D}=\mathcal{R}^{C H S}$ and then using an extension argument to show that the axioms are also incompatible on any larger domain.

\footnotetext{
${ }^{9}$ Moulin and Thomson [12] have considered a related axiom. Their axiom requires each person to be provided with a commodity bundle that is weakly preferred to having an $\varepsilon$ share of the aggregate endowment, where $0<\varepsilon \leq 1 / n$.
} 
Our impossibility theorem can be used to provide an alternative proof of a slight variant of one of the main results in Serizawa [19]. In Serizawa's theorem, endowments are privately owned. He has shown that for a preference domain satisfying the restriction in our theorem, it is not possible to satisfy strategy-proofness, Pareto optimality, and individual rationality.

Corollary. Suppose that endowments are privately owned with $\omega^{i} \gg 0_{m}$ for all $i \in N$ and that $\mathcal{R}^{C H S} \subseteq \mathcal{D} \subseteq \mathcal{R}$. If a social choice function $f: \mathcal{D}^{n} \rightarrow X$ satisfies strategy-proofness and Pareto optimality, then it violates individual rationality. ${ }^{10}$

Proof. First, suppose that $f$ is strategy-proof and Pareto optimal and that $\mathcal{R}^{C H S} \subseteq \mathcal{D} \subseteq \mathcal{R}^{C}$. With this domain restriction, we show that individual rationality implies minimum consumption guarantee when $\omega^{i} \gg 0_{m}$ for all $i \in N$, which contradicts the Theorem. ${ }^{11}$

Let $\bar{\varepsilon}=\min _{i \in N} \min _{k \in M} \omega_{k}^{i}$. By assumption, $\bar{\varepsilon}>0$. Because preferences are continuous on $\mathbb{R}_{+}^{m}$ and strictly monotonic on $\mathbb{R}_{++}^{m}$, individual rationality implies that for any $\mathbf{R} \in \mathcal{D}^{n}$ and any $i \in N,\left\|f^{i}(\mathbf{R})\right\| \geq \min _{k \in M} \omega_{k}^{i} \geq \bar{\varepsilon}$. Hence, $f$ satisfies minimum consumption guarantee.

Having established the theorem for classical domains, an extension argument similar to the one used in part (b) of the proof of the Theorem can be used to establish the theorem when $\mathcal{R}^{C} \subset \mathcal{D} \subseteq \mathcal{R}$.

\section{Preliminary Results}

In this section, we present a number of preliminary results that are used to help prove the Theorem. With the exception of Lemma 7, the lemmas in this section have already been established in the earlier literature or are minor extensions of known results. See Serizawa [19] for further details.

One implication of stategy-proofness when individuals have classical preferences is that if an individual's consumption of some good increases as a result of a change in this person's reported preference, then the consumption of at least one other good must decrease. A social choice function with this property is said to be diagonal. See Barberà and Jackson [1], Serizawa [19], and Zhou [21].

\footnotetext{
${ }^{10}$ Serizawa [19] only requires that $\omega^{i}>0_{m}$ for all $i \in N$.

${ }^{11}$ Individual rationality does not imply minimum consumption guarantee if individuals do not have positive endowments of all goods.
} 
Lemma 1. Suppose that $\mathcal{D} \subseteq \mathcal{R}^{C}$ and $f: \mathcal{D}^{n} \rightarrow X$ is a strategy-proof social choice function. For all $\mathbf{R} \in \mathcal{D}^{n}$, all $i \in N$, and all $\bar{R}^{i} \in \mathcal{D}$, if $\mathbf{x} \in X$ is such that $x^{i}<f^{i}(\mathbf{R})$ or $x^{i}>f^{i}(\mathbf{R})$, then $\mathbf{x} \neq f\left(\bar{R}^{i} ; \mathbf{R}^{-i}\right)$.

Consider a preference $R \in \mathcal{R}$ and a consumption bundle $x \in \mathbb{R}_{+}^{m}$. A preference $\bar{R} \in \mathcal{R}$ is a (strict) Maskin monotonic transform of $R$ at $x$ (see Maskin [11]) if $[\bar{x} \in U C(\bar{R}, x)$ and $\bar{x} \neq x] \rightarrow \bar{x} P x$.

If an individual receives the commodity bundle $x$ at the profile $\mathbf{R}$, strategyproofness implies that this individual receives the same commodity bundle if his preference is subjected to a Maskin monotonic transform at $x$.

Lemma 2. Suppose that $f: \mathcal{D}^{n} \rightarrow X$ is a strategy-proof social choice function. For all $\mathbf{R} \in \mathcal{D}^{n}$ and all $i \in N$, if $\bar{R}^{i} \in \mathcal{D}$ is a Maskin monotonic transform of $R^{i}$ at $f^{i}(\mathbf{R})$, then $f^{i}\left(\bar{R}^{i} ; \mathbf{R}^{-i}\right)=f^{i}(\mathbf{R})$.

Lemma 2 is a private goods counterpart to a public goods theorem established by Muller and Satterthwaite [13]. A proof of Lemma 2 may be found in Serizawa [19]. Note that Lemma 2 holds for any domain. If there are only two individuals, an implication of this lemma is that nobody's consumption bundle is changed if a Maskin monotonic transform is applied to one person's preference at the commodity bundle assigned to him by the social choice function. With more than two individuals, the commodity bundles of the other individuals can be affected by this kind of change in $i$ 's preference.

A preference $R$ on $\mathbb{R}_{+}^{m}$ is a Cobb-Douglas preference if it can be represented by a utility function $U: \mathbb{R}_{+}^{m} \rightarrow \mathbb{R}$ of the form

$$
U(x)=x_{1}^{\alpha_{1}} \cdots x_{m}^{\alpha_{m}}
$$

for all $x \in \mathbb{R}_{+}^{m}$, where $\alpha_{k}>0$ for all $k \in M$. A preference $R$ on $\mathbb{R}_{+}^{m}$ is a constant-elasticity-of-substitution (CES) preference if it can be represented by a utility function $U: \mathbb{R}_{+}^{m} \rightarrow \mathbb{R}$ of the form

$$
U(x)=\left[\sum_{k \in M} b_{k}\left(x_{k}\right)^{\rho}\right]^{1 / \rho}
$$

for all $x \in \mathbb{R}_{+}^{m}$, where $b_{k}>0$ for all $k \in M$ and $0 \neq \rho<1$. Cobb-Douglas and CES preferences are in $\mathcal{R}^{C H S}$. Given $x^{0} \in \mathbb{R}_{++}^{m}$, the preference $R$ on $\mathbb{R}_{+}^{m}$ is the $x^{0}$-generated Leontief preference if it can be represented by a utility function $U: \mathbb{R}_{+}^{m} \rightarrow \mathbb{R}$ of the form

$$
U(x)=\min \left\{x_{1} / x_{1}^{0}, \ldots, x_{m} / x_{m}^{0}\right\}
$$


for all $x \in \mathbb{R}_{+}^{m}$. Given $p \in \mathbb{R}_{++}^{m}$, the preference $R$ on $\mathbb{R}_{+}^{m}$ is the linear preference with normal $p$ if it can be represented by a utility function $U: \mathbb{R}_{+}^{m} \rightarrow \mathbb{R}$ of the form

$$
U(x)=\sum_{k \in M} p_{k} x_{k}
$$

for all $x \in \mathbb{R}_{+}^{m}$.

For any smooth classical preference $R$ on $\mathbb{R}_{+}^{m}$ and any consumption bundle $x^{0}$ in the interior of the consumption set, let $U$ be a utility representation of $R$ that is differentiable at $x^{0}$. Let $U^{\rho}$ be the utility function obtained by setting

$$
b_{k}=\frac{\partial U\left(x^{0}\right)}{\partial x_{k}}\left(x_{k}^{0}\right)^{1-\rho}
$$

in (2). It is readily verified that $\nabla U^{\rho}\left(x^{0}\right)$ (the gradient of $U^{\rho}$ at $x^{0}$ ) is proportional to $\nabla U\left(x^{0}\right)$ when $0 \neq \rho<1$. As $\rho$ approaches $1, U^{\rho}$ approaches a linear utility function whose indifference contours are orthogonal to $\nabla U\left(x^{0}\right)$. In the limit as $\rho$ goes to $-\infty, U^{\rho}$ represents the $x^{0}$-generated Leontief preference. Hence, for sufficiently small $\rho, U^{\rho}$ represents a preference that is a CES Maskin monotonic transform of $R$ at $x^{0}$. Further, this transformed preference can be chosen to be arbitrarily close to the $x^{0}$-generated Leontief preference.

Lemma 3. If $R \in \mathcal{R}^{C S}$ and $x^{0} \in \mathbb{R}_{++}^{m}$, then there exists a CES Maskin monotonic transform of $R$ at $x^{0}$ that is arbitrarily close to the $x^{0}$-generated Leontief preference. ${ }^{12}$

Pareto optimality implies that individuals with identical preferences are assigned commodity bundles that are proportional to each other by the social choice function if their preferences are classical and homothetic. See Schummer [17] and Serizawa [19]. In the differentiable case, this follows because with a strictly convex, homothetic preference, the marginal rates of substitution for two commodity bundles are equal if and only if the commodity bundles are proportional.

Lemma 4. Suppose that $f: \mathcal{D}^{n} \rightarrow X$ is a social choice function satisfying Pareto optimality. For all $\mathbf{R} \in \mathcal{D}^{n}$ and all $i, j \in N$, if $R^{i}=R^{j} \in \mathcal{R}^{C H}$ and $f^{i}(\mathbf{R}) \neq 0_{m}$, then there exists a $\lambda \geq 0$ such that $f^{j}(\mathbf{R})=\lambda f^{i}(\mathbf{R})$.

\footnotetext{
${ }^{12} \mathrm{~A}$ natural metric for measuring the closeness of continuous, monotonic preferences is the Kannai [7] metric.
} 
Serizawa [19] has shown that if all but one person shares a common classical, homothetic preference, from the perspective of the remaining individual, we effectively have a two-person economy for the purposes of identifying Pareto-optimal allocations. More precisely, for an economy with endowment $\Omega$, if one individual, say person 1 , has a classical preference $R^{1}$ and everyone else has the same classical, homothetic preference $R^{0}$, then the projection of the set of Pareto optimal allocations to 1's consumption set in the $n$-person economy is the same as the set of Pareto optimal allocations (viewed from person 1's perspective) for the two-person economy defined by the profile $\left(R^{1}, R^{0}\right)$ and the endowment $\Omega$.

To state Serizawa's result formally, we need to introduce some more notation. Given $\Omega \in \mathbb{R}_{+}^{m}$, let

$$
X_{2}=\left\{\mathbf{x} \in \mathbb{R}_{+}^{2 m} \mid x^{1}+x^{2} \leq \Omega\right\}
$$

denote the set of feasible allocations for an exchange economy with two individuals and endowment $\Omega$ and let $X_{2}^{*}=X_{2} \cap \mathbb{R}_{++}^{2 m}$. The set of Pareto optimal allocations for this economy is $\mathcal{P}_{2}\left(\left(R^{1}, R^{2}\right), \Omega\right)$ when the profile is $\left(R^{1}, R^{2}\right) \in \mathcal{D}^{2}$. For all $A \subseteq X$ and all $A \subseteq X_{2}$, the projection of $A$ on $i$ 's consumption set is $\operatorname{Pr}_{i} A$.

Lemma 5. For all $\Omega \in \mathbb{R}_{++}^{m}$, all $R^{0} \in \mathcal{R}^{C H}$, all $i \in N$, and all $R^{i} \in \mathcal{R}^{C}$, $\operatorname{Pr}_{i} \mathcal{P}_{2}\left(\left(R^{i} ; R^{0}\right), \Omega\right)=\operatorname{Pr}_{i} \mathcal{P}\left(\left(R^{i} ; R^{0}, \ldots, R^{0}\right), \Omega\right)$.

Now suppose that the social choice function satisfies strategy-proofness, Pareto optimality, and minimum consumption guarantee. Consider a profile $\mathbf{R}$ in which everybody has classical, smooth, and homothetic preferences and in which all but one individual, say person 1, has the same preference $R^{0}$. Suppose that individual 1's consumption bundle at this profile is in $X^{*}$ and is not proportional to $\Omega$. We know from Lemma 4 that if $\bar{R}^{0}$ is a classical, homothetic, Maskin monotonic transform of $R^{0}$ at the aggregate consumption bundle of individuals 2 through $n$, then it is also a Maskin monotonic transform of $R^{0}$ at the assigned consumption bundles of each of these individuals at the profile $\mathbf{R}$. If person $j$ 's preference $(j \neq 1)$ is changed from $R^{0}$ to $\bar{R}^{0}$, Lemma 2 implies that $j$ 's consumption doesn't change, but, in general, we don't know what happens to the consumption of the other individuals when $n>2$. Lemma 6 shows that if $R^{0}$ is changed to $\bar{R}^{0}$ for all $j \neq 1$, then person 1's consumption is unaffected if the Maskin monotonic transform $\bar{R}^{0}$ is smooth, not just classical and homothetic. 
Lemma 6. Suppose that $f: \mathcal{D}^{n} \rightarrow X$ is a strategy-proof social choice function that satisfies Pareto optimality and minimum consumption guarantee. For all $i \in N$ and all $R^{i}, R^{0}, \bar{R}^{0} \in \mathcal{D} \cap \mathcal{R}^{C H S}$, if $0_{m} \ll f^{i}\left(R^{i} ; R^{0}, \ldots, R^{0}\right) \ll$ $\Omega, f^{i}\left(R^{i} ; R^{0}, \ldots, R^{0}\right)$ is not proportional to $\Omega$, and $\bar{R}^{0}$ is a Maskin monotonic transform of $R^{0}$ at $\sum_{j \neq i} f^{j}\left(R^{i} ; R^{0}, \ldots, R^{0}\right)$, then $f^{i}\left(R^{i} ; R^{0}, \ldots, R^{0}\right)=$ $f^{i}\left(R^{i} ; \bar{R}^{0}, \ldots, \bar{R}^{0}\right)$.

Lemma 6 is another application of the observation that if all but one person has the same classical, homothetic preference, then, in some circumstances, this group of individuals can be treated as an aggregate from the perspective of the other individual. Note that it is not claimed in Lemma 6 that the individual consumptions of individuals other than $i$ are unaffected by the preference change.

Lemma 6 is a variant of a theorem established by Serizawa [19]. Serizawa assumes that $\bar{R}^{0}$ is a CES preference, but all that is required for his proof is that it be in $\mathcal{R}^{C H S}$. In addition, Serizawa assumes that the social choice function satisfies individual rationality, rather than minimum consumption guarantee. However, individual rationality is only used by Serizawa to show that nobody receives the consumption bundle $0_{m}$ at any of the profiles considered in the proof of his theorem. This conclusion is also implied by our minimum consumption guarantee axiom.

A preference $R$ on $\mathbb{R}_{+}^{m}$ is additively separable if there exist functions $f^{k}: \mathbb{R}_{+} \rightarrow \mathbb{R}$ for all $k \in M$ such that $R$ can be represented by a utility function $U: \mathbb{R}_{+}^{m} \rightarrow \mathbb{R}$ of the form

$$
U(x)=\sum_{k \in M} f^{k}\left(x_{k}\right)
$$

for all $x \in \mathbb{R}_{+}^{m}$. A smooth, additively separable preference has the property that the marginal rate of substitution between a pair of goods only depends on the consumptions of these two goods. A smooth preference $R$ on $\mathbb{R}_{+}^{m}$ is pairwise homothetic if for all $x, x^{\prime} \in \mathbb{R}_{++}^{m}$, all $k, k^{\prime} \in M$, and all $\lambda>0$, $\left[x_{j}^{\prime}=\lambda x_{j}\right.$ for $j=k, k^{\prime}$ and $x_{j}^{\prime}=x_{j}$ for $\left.j \neq k, k^{\prime}\right] \rightarrow \operatorname{MRS}_{k k^{\prime}}(x, R)=$ $\operatorname{MRS}_{k k^{\prime}}\left(x^{\prime}, R\right)$, where $\operatorname{MRS}_{k k^{\prime}}(x, R)$ denotes the marginal rate of substitution between goods $k$ and $k^{\prime}$ at the commodity bundle $x$ for the preference $R$. Note that Cobb-Douglas and CES preferences are additively separable and pairwise homothetic.

A set of allocations $A \subset X$ is increasing from person i's perspective at $x^{i} \in \operatorname{Pr}_{i} A$ if for all $\bar{x}^{i} \in \operatorname{Pr}_{i} A$ and all $k, k^{\prime} \in M, x_{k}^{i}<\bar{x}_{k}^{i} \leftrightarrow x_{k^{\prime}}^{i}<\bar{x}_{k^{\prime}}^{i}$. 
Consider a two-person economy in which both individuals have smooth classical preferences that are also additively separable and pairwise homothetic. Lemma 7 demonstrates that the set of Pareto optimal allocations for this economy is increasing from the perspective of either individual at a Pareto optimal allocation with positive consumptions.

Lemma 7. For all $\Omega \in \mathbb{R}_{++}^{m}$, all $R^{1}, R^{2} \in \mathcal{R}^{C S}$ that are additively separable and pairwise homothetic, all $i \in\{1,2\}$, and all $x^{i} \in \operatorname{Pr}_{i}\left[\mathcal{P}_{2}\left(\left(R^{1}, R^{2}\right), \Omega\right) \cap X_{2}^{*}\right]$, $\operatorname{Pr}_{i} \mathcal{P}_{2}\left(\left(R^{1}, R^{2}\right), \Omega\right)$ is increasing from person $i$ 's perspective at $x^{i}$.

Proof. Without loss of generality, let $i=1$.

First, suppose that $m=2$. Consider any $x^{1} \in \operatorname{Pr}_{1}\left[\mathcal{P}_{2}\left(\left(R^{1}, R^{2}\right), \Omega\right) \cap X_{2}^{*}\right]$. Pareto optimality requires that $\operatorname{MRS}_{12}\left(x^{1}, R^{1}\right)=\operatorname{MRS}_{12}\left(\Omega-x^{1}, R^{2}\right)$. If $x^{1}$ is proportional to $\Omega$, pairwise homotheticity implies that $\operatorname{Pr}_{i} \mathcal{P}_{2}\left(\left(R^{1}, R^{2}\right), \Omega\right)$ is the line segment joining the origin of 1 's consumption set to $\Omega$, from which the conclusion follows immediately. If $x^{1}$ is not proportional to $\Omega$, we can without loss of generality suppose that $x_{1}^{1} / x_{2}^{1}>\Omega_{1} / \Omega_{2}$. Let $L^{1}$ be the restriction to $\operatorname{Pr}_{1} X_{2}$ of the line that includes $0_{2}$ and $x^{1}$ and let $L^{2}$ be the restriction to $\operatorname{Pr}_{1} X_{2}$ of the line that includes $\Omega$ and $x^{1}$. See Figure 1. By pairwise homotheticity, $\operatorname{MRS}_{12}\left(\bar{x}^{1}, R^{1}\right)=\operatorname{MRS}_{12}\left(x^{1}, R^{1}\right)$ for all $\bar{x}^{1} \in L_{1} \backslash\left\{0_{2}\right\}$ and $\operatorname{MRS}_{12}\left(\Omega-\bar{x}^{1}, R^{2}\right)=\operatorname{MRS}_{12}\left(\Omega-x^{1}, R^{2}\right)$ for all $\bar{x}^{1} \in L_{2} \backslash\{\Omega\}$. Because preferences are strictly convex, the marginal rates of substitution can only be equal in the two regions between $L_{1}$ and $L_{2}$ in Figure 1 . Thus, in $X_{2}^{*}$, any Pareto optimal allocation lies in one of these two regions. Similarly, on the boundary of the Edgeworth box, an allocation $\left(\bar{x}^{1}, \Omega-\bar{x}^{1}\right)$ can only satisfy the necessary conditions for Pareto optimality if either (i) $\bar{x}_{2}^{1}=0$ and $\bar{x}^{1}$ lies to the left of $L_{2}$ or (ii) $\bar{x}_{1}^{1}=\Omega_{1}$ and $\bar{x}^{1}$ lies above $L^{1}$. Because $L_{1}$ and $L_{2}$ have positive slopes, it follows that $\operatorname{Pr}_{1} \mathcal{P}_{2}\left(\left(R^{1}, R^{2}\right), \Omega\right)$ is increasing from person 1's perspective at $x^{1}$.

Now, suppose that that $m>2$. Consider any $x^{1} \in \operatorname{Pr}_{1}\left[\mathcal{P}_{2}\left(\left(R^{1}, R^{2}\right), \Omega\right) \cap\right.$ $\left.X_{2}^{*}\right]$. Pareto optimality requires that $\operatorname{MRS}_{1 k}\left(x^{1}, R^{1}\right)=\operatorname{MRS}_{1 k}\left(\Omega-x^{1}, R^{2}\right)$ for all $k \neq 1$. Fix the consumptions of all goods but 1 and $k$ for an arbitrary $k \neq 1$. Because the preferences are pairwise homothetic, the proof of the two-good case shows that if $x_{1}^{1}$ is increased (resp. decreased), the first-order conditions for Pareto optimality for goods 1 and $k$ of the two individuals can only be satisfied if $x_{k}^{1}$ is also increased (resp. decreased). We now adjust the consumptions of the other goods so that the allocation is Pareto optimal. Because preferences are additively separable, the marginal rates of substitution for goods 1 and $k$ are independent of the quantities consumed of the 
other goods. Hence, if $\bar{x}^{1} \in \operatorname{Pr}_{1} \mathcal{P}_{2}\left(\left(R^{1}, R^{2}\right), \Omega\right)$ and $\bar{x}_{1}^{1}>x_{1}^{1}\left(\right.$ resp. $\left.\bar{x}_{1}^{1}<x_{1}^{1}\right)$, then $\bar{x}_{k}^{1}>x_{k}^{1}\left(\operatorname{resp} . \bar{x}_{k}^{1}<x_{k}^{1}\right)$ for all $k \neq 1$.

\section{A Proof of the Theorem}

In this section, we provide a proof of the Theorem. In part (a) of the proof, the Theorem is established for the domain $\left(\mathcal{R}^{C H S}\right)^{n}$. In part (b), an extension argument is used to show that the Theorem also holds on larger domains.

(a) In this part of the proof, it is supposed that $\mathcal{D}=\mathcal{R}^{C H S}$. Contrary to the theorem, suppose that $f$ is a strategy-proof social choice function that satisfies Pareto optimality and minimum consumption guarantee. Because $f$ guarantees minimum consumption, there exists an $\varepsilon>0$ such that for all $\mathbf{R} \in \mathcal{D}^{n}$ and all $i \in N,\left\|f^{i}(\mathbf{R})\right\| \geq \varepsilon$. Consider any such $\varepsilon$ and let $d=\left(d_{1}, \ldots, d_{m}\right) \in \mathbb{R}_{++}^{m}$ be the commodity bundle proportional to $\Omega$ that is $\varepsilon$ distance from $0_{m}$.

Step 1. Given $\alpha \geq 1$, define the Cobb-Douglas utility function $U^{\alpha}$ on $\mathbb{R}_{+}^{m}$ by setting

$$
U^{\alpha}(x)=x_{1}^{\alpha} x_{2} \cdots x_{m}
$$

for all $x \in \mathbb{R}_{+}^{m}$. Let $R(\alpha)$ be the preference represented by $U^{\alpha}$.

First, suppose that everyone has the symmetric Cobb-Douglas preference $R(1)$. Let $\mathbf{x}^{\circ}=f(R(1), \ldots, R(1))$. By minimum consumption guarantee and Lemma $4, x^{\circ i}$ is proportional to $\Omega$ for all $i \in N$. Clearly, there exists an $i \in N$ such that $x^{\circ i} \leq \Omega / n$. Without loss of generality, we assume that person 1 is such an individual.

Now, suppose that person 1 has the preference $R(\alpha)$ for some $\alpha>1$. Let $\mathbf{x}^{\alpha}=f(R(\alpha), R(1), \ldots, R(1))$. By minimum consumption guarantee, $x^{\alpha i} \neq 0_{m}$ for all $i \in N$. Lemma 4 then implies that $x^{\alpha i}$ is proportional to $x^{\alpha j}$ for all $i, j \neq 1$. Because everyone has a Cobb-Douglas preference, Pareto optimality implies that $\mathbf{x}^{\alpha} \in X^{*}$.

By Lemma 5, $\operatorname{Pr}_{1} \mathcal{P}_{2}((R(\alpha), R(1)), \Omega)=\operatorname{Pr}_{1} \mathcal{P}((R(\alpha), R(1), \ldots, R(1)), \Omega)$. For allocations in the interior of $X$, Pareto optimality requires that marginal rates of substitution be equalized. In particular,

$$
\frac{\alpha x_{k}^{\alpha 1}}{x_{1}^{\alpha 1}}=\frac{\Omega_{k}-x_{k}^{\alpha 1}}{\Omega_{1}-x_{1}^{\alpha 1}}, \quad \forall k \in M \backslash\{1\},
$$

where use has been made of the fact that everyone but person 1 has the same homothetic preference and has consumption bundles that are proportional 
to each other. For given values of the consumption of good 1, everyone has symmetric Cobb-Douglas preferences for the other goods. Hence, by Lemma 4, Pareto optimality also implies that

$$
\frac{x_{k^{\prime}}^{\alpha 1}}{x_{k}^{\alpha 1}}=\frac{\Omega_{k^{\prime}}}{\Omega_{k}}=\frac{x_{k^{\prime}}^{\circ 1}}{x_{k}^{\circ 1}}, \quad \forall k, k^{\prime} \in M \backslash\{1\} .
$$

By Lemma 1, neither $x^{\alpha 1}>x^{\circ 1}$ nor $x^{\alpha 1}<x^{\circ 1}$ holds. From (8) and (9), it then follows that $x^{\alpha 1} \in\left(x_{1}^{\circ 1}, \Omega_{1}\right) \times \prod_{k \neq 1}\left(0, x_{k}^{\circ 1}\right)$.

Other than requiring $\alpha>1$, the value of $\alpha$ in this step is arbitrary. If there exists an $\alpha$ for which $x_{k}^{\alpha 1}<d_{k}$ for all $k \neq 1$, say $\alpha=\bar{\alpha}$, proceed to Step 5. Otherwise, go to Step 2.

Step 2. Let $H^{\alpha}$ denote the hyperplane through $x^{\alpha 1}$ whose normal is given by $\nabla U^{\alpha}\left(x^{\alpha 1}\right)$. We want to determine the value of $a_{1}$ for which $a=$ $\left(a_{1}, d_{2}, x_{3}^{\alpha 1}, \ldots, x_{m}^{\alpha 1}\right) \in H^{\alpha}$. Using (8), it is straightforward to show that

$$
a_{1}=x_{1}^{\alpha 1}+\left[\frac{\Omega_{1}-x_{1}^{\alpha 1}}{\Omega_{2}-x_{2}^{\alpha 1}}\right]\left[x_{2}^{\alpha 1}-d_{2}^{1}\right] .
$$

Let $\gamma=\Omega_{1}-x_{1}^{\alpha 1}$, which we know is positive. Recall that $x_{2}^{\alpha 1}<x_{2}^{\circ 1} \leq \Omega_{2} / n$ and $d_{2}^{1}>0$. From (10), we have

$$
\begin{aligned}
a_{1} & <\Omega_{1}-\gamma+\gamma\left[\frac{\frac{\Omega_{2}}{n}-d_{2}^{1}}{\Omega_{2}-\frac{\Omega_{2}}{n}}\right] \\
& <\Omega_{1}-\gamma+\gamma\left[\frac{\frac{\Omega_{2}}{n}}{\Omega_{2}-\frac{\Omega_{2}}{n}}\right] \\
& =\Omega_{1}-\gamma+\gamma\left[\frac{1}{n-1}\right] \\
& =\Omega_{1}-\gamma\left[\frac{n-2}{n-1}\right] \\
& \leq \Omega_{1},
\end{aligned}
$$

where the last inequality follows from the fact that $n \geq 2$ and $\gamma>0$. Thus, $a_{1}<\Omega_{1}$.

From the discussion in Section 4, we know that we can find a CES preference $R^{\prime}$ on $\mathbb{R}_{+}^{m}$ and associated utility function $U^{\prime}$ for which (i) $\nabla U^{\prime}\left(x^{\alpha 1}\right)$ is proportional to $\nabla U^{\alpha}\left(x^{\alpha 1}\right)$ and (ii) $R^{\prime}$ is arbitrarily close to the linear preference whose indifference contours are hyperplanes parallel to $H^{\alpha}$. In view of 
the discussion in the preceding paragraph, $R^{\prime}$ can be chosen so that $x^{\alpha 1} I^{\prime} \bar{a}$, where $\bar{a}=\left(\bar{a}_{1}, d_{2}, x_{3}^{\alpha 1}, \ldots, x_{3}^{\alpha 1}\right)$ for some $\bar{a}_{1} \in\left(a_{1}, \Omega_{1}\right)$.

Step 3. Let $\mathbf{x}^{\prime}=f\left(R^{\prime}, R(1), \ldots, R(1)\right)$. We now show that $x^{1}=x^{\alpha 1}$.

Because $f$ is Pareto optimal and $f^{1}(R(\alpha), R(1), \ldots, R(1))=x^{\alpha 1}$, by the construction of $R^{\prime}$, the gradients $\nabla U^{\prime}\left(x^{\alpha 1}\right), \nabla U^{\alpha}\left(x^{\alpha 1}\right)$, and $\nabla U^{1}\left(\Omega-x^{\alpha 1}\right)$ are all proportional to each other. Thus, the marginal rates of substitution of $R^{\prime}$ at $x^{\alpha 1}$ are equal to the marginal rates of substitution of $R(1)$ at $\Omega-$ $x^{\alpha 1}$. Because preferences are convex and $\left(x^{\alpha 1}, \Omega-x^{\alpha 1}\right) \in X_{2}^{*}$, it follows that $x^{\alpha 1} \in \operatorname{Pr}_{1} \mathcal{P}_{2}\left(\left(R^{\prime}, R(1)\right), \Omega\right)$. Because $f$ is Pareto optimal, we also have $x^{\prime 1} \in \operatorname{Pr}_{1} \mathcal{P}\left(\left(R^{\prime}, R(1), \ldots, R(1)\right), \Omega\right)$ and, hence, by Lemma 5 that $x^{\prime 1} \in$ $\operatorname{Pr}_{1} \mathcal{P}\left(\left(R^{\prime}, R(1)\right), \Omega\right)$.

Suppose that $x^{1} \neq x^{\alpha 1}$. Because CES and Cobb-Douglas preferences are additively separable and pairwise homothetic and because $\left(x^{\alpha 1}, \Omega-x^{\alpha 1}\right) \in$ $X_{2}^{*}$, by Lemma 7 , either $x^{\alpha 1} \ll x^{1}$ or $x^{\alpha 1} \gg x^{11}$. In either case, we have a contradiction with Lemma 1. Thus, $x^{1}=x^{\alpha 1}$.

Step 4 . Let $\tilde{R}(\nu)$ be the preference represented by a CES utility function $\tilde{U}^{\nu}$ on $\mathbb{R}_{+}^{m}$ of the form

$$
\tilde{U}^{\nu}(x)=\left[\nu \bar{b}_{1}\left(x_{1}\right)^{\rho}+\sum_{k=2}^{m} \nu^{-1} \bar{b}_{k}\left(x_{k}\right)^{\rho}\right]^{1 / \rho}
$$

for all $x \in \mathbb{R}_{+}^{m}$, where $\left(\bar{b}_{1}, \ldots, \bar{b}_{m}\right) \in \mathbb{R}_{++}^{m}$ and $\rho$ are chosen so that $\tilde{R}(1)=R^{\prime}$. Let $Y=\left\{\mathbf{x} \in X \mid\right.$ (i) $\sum_{i \in N} x^{i}=\Omega$ and (ii) $x_{1}^{1}=\Omega_{1}$ or $\left.x_{2}^{1}=\cdots=x_{m}^{1}=0\right\}$. As $\nu \rightarrow \infty$ in (11), person 1's preference approaches the preference $R(\infty)$ in which he only cares about good 1 . In the limit, the set of Pareto optimal allocations $\mathcal{P}((\tilde{R}(\infty), R(1), \ldots, R(1)), \Omega)$ is contained in $Y$. Thus, because $\bar{a}_{1}<\Omega_{1}$, there exists a $\bar{\nu}$ sufficiently large such that for all $x^{1} \in L C\left(R^{\prime}, x^{\alpha 1}\right) \cap$ $U C\left(\tilde{R}, x^{\alpha 1}\right) \cap \operatorname{Pr}_{1} \mathcal{P}((\tilde{R}, R(1), \ldots, R(1)), \Omega)$, we have $d_{1}<x_{1}^{\alpha 1}<x_{1}^{1}<\Omega_{1}$ and $0<x_{k}^{1}<d_{k}$ for all $k \neq 1$, where $\tilde{R}=\tilde{R}(\bar{\nu})$. See Figure $2 .{ }^{13}$

Let $\tilde{\mathbf{x}}=f(\tilde{R}, R(1), \ldots, R(1))$. Strategy-proofness implies that $\tilde{x}^{1} \in$ $L C\left(R^{\prime}, x^{\alpha 1}\right) \cap U C\left(\tilde{R}, x^{\alpha 1}\right)$. Because $f$ is Pareto optimal, we also have $\tilde{x}^{1} \in$ $\operatorname{Pr}_{1} \mathcal{P}((\tilde{R}, R(1), \ldots, R(1)), \Omega)$. Hence, $d_{1}<\tilde{x}_{1}^{1}<\Omega_{1}$ and $0<\tilde{x}_{k}^{1}<d_{k}$ for all $k \neq 1$.

Step 5. If Steps 2, 3, and 4 are utilized, let $R^{*}=\tilde{R}$ and $\mathbf{x}^{*}=\tilde{\mathbf{x}}$. Otherwise, let $R^{*}=R(\bar{\alpha})$ and $\mathbf{x}^{*}=\mathbf{x}^{\bar{\alpha}}$. In either case, $d_{1}<x_{1}^{* 1}<\Omega_{1}$ and $0<x_{k}^{* 1}<d_{k}$ for all $k \neq 1$. These inequalities imply that there exists a $p \in \mathbb{R}_{++}^{m}$ such

\footnotetext{
${ }^{13}$ In Figure 2, the curve through $\tilde{x}^{1}$ is $\operatorname{Pr}_{1} \mathcal{P}_{2}((\tilde{R}, R(1)), \Omega)$.
} 
that $\sum_{k \in M} p_{k} d_{k}>\sum_{k \in M} p_{k} x_{k}^{* 1}$. For all $k \in M$, let $e^{k}$ be the vector for which $e_{k}^{k}=1$ and $e_{k^{\prime}}^{k}=0$ for all $k^{\prime} \neq k$.

Because a CES preference can be chosen to be arbitrarily close to a linear preference, we can find a CES preference $\bar{R}$ on $\mathbb{R}_{+}^{m}$ and associated utility function $\bar{U}$ with the following properties: (i) $\nabla \bar{U}\left(x^{* 1}\right)=p$, (ii) $d \bar{P} x^{* 1}$, (iii) there exists an open, convex cone $K_{p} \subset \mathbb{R}_{++}^{m}$ with vertex at $0_{m}$ containing $p$ such that $\nabla \bar{U}\left(x^{1}\right) \in K_{p}$ for all $x^{1}$ in $\operatorname{Pr}_{1} X$, and (iv) for all $k \in M$, there exists an open, convex cone $K_{k} \subset \mathbb{R}^{m}$ with vertex at $0_{m}$ containing $e^{k}$ such that $K_{p} \cap K_{k}=\emptyset$. Note that (iii) and (iv) imply that for any pair of goods $k, k^{\prime} \in M$, there is an open interval $\mathcal{I}_{k k^{\prime}}$ whose lower bound is strictly positive and whose upper bound is finite such that $\left|\mathrm{MRS}_{k k^{\prime}}\left(x^{1}, \bar{R}\right)\right| \in \mathcal{I}_{k k^{\prime}}$ for all $x^{1} \in \operatorname{Pr}_{1} X$.

Step 6. We now construct a CES Maskin monotonic transform of $R(1)$ at $\sum_{i \neq 1} x^{* i}=\Omega-x^{* 1}$. For $x \in \mathbb{R}^{m}$ and $\delta>0$, let $B_{\delta}(x)$ be the open ball of radius $\delta$ centered at $x$. We choose $\bar{\delta}>0$ sufficiently small so that (i) $U C(\bar{R}, d) \cap B_{\bar{\delta}}\left(x^{* 1}\right)=\emptyset$ and (ii) there exists a $\delta^{*}>\bar{\delta}$ for which $B_{\delta^{*}}\left(x^{* 1}\right) \subset$ $\prod_{k \in M}\left(0, \Omega_{k}\right)$. Let $Z=\left\{x \in \mathbb{R}_{+}^{m} \mid x_{k}=\Omega_{k}-x_{k}^{* 1}\right.$ for some $\left.k \in M\right\}$. Let $K^{*} \subset \mathbb{R}_{++}^{m}$ be an open, convex cone with vertex at $0_{m}$ containing $\Omega-x^{* 1}$ for which $\left(K^{*} \cap Z\right) \subset B_{\bar{\delta}}\left(\Omega-x^{* 1}\right)$ and $\Omega \notin K^{*}$. Because $K^{*}$ is a cone, $\left(K^{*} \cap Z\right) \subset B_{\bar{\delta}}\left(\Omega-x^{* 1}\right)$ and $U C(\bar{R}, d) \cap B_{\bar{\delta}}\left(x^{* 1}\right)=\emptyset$ imply that $x^{1} \gg x^{* 1}$ for all $x^{1} \in\left(\Omega-K^{*}\right) \cap U C(\bar{R}, d)$.

By Lemma 3, we can find a CES Maskin monotonic transform $\hat{R}$ of $R(1)$ at $\Omega-x^{* 1}$ that is arbitrarily close to the $\left(\Omega-x^{* 1}\right)$-generated Leontief preference. Let $\hat{U}$ be a differentiable utility function representing $\hat{R}$. We choose $\hat{R}$ so that for all $x \in \mathbb{R}_{+}^{m} \backslash K^{*}$ for which $x \neq 0_{m}, \nabla \hat{U}(x) \notin K_{p}$. Because $\hat{R}$ is a CES Maskin monotonic transform of $R(1)$ at $\Omega-x^{* 1}$, by Lemma 6 , $f^{1}\left(R^{*}, \hat{R}, \ldots, \hat{R}\right)=x^{* 1}$.

Step $7 .{ }^{14}$ Let $\hat{\mathbf{x}}=f(\hat{R}, \ldots, \hat{R})$. By minimum consumption guarantee and Lemma $4, \hat{x}^{i}$ is proportional to $\Omega$ and $\hat{x}^{i} \geq d$ for all $i \in N$.

Because $\hat{x}^{1} \geq d, U C\left(\bar{R}, \hat{x}^{1}\right) \subseteq U C(\bar{R}, \bar{d})$. By Step 6 we know that $x^{1} \gg$ $x^{* 1}$ for all $x^{1} \in\left(\Omega-K^{*}\right) \cap U C(\bar{R}, d)$. Hence, $x^{1} \gg x^{* 1}$ for all $x^{1} \in(\Omega-$ $\left.K^{*}\right) \cap U C\left(\bar{R}, \hat{x}^{1}\right)$.

Step 8. Let $\overline{\mathbf{x}}=f(\bar{R}, \hat{R}, \ldots, \hat{R})$. Comparing the outcomes when person 1 reports either $\bar{R}$ or $\hat{R}$ and everyone else reports $\hat{R}$, strategy-proofness implies that $\bar{x}^{1} \in U C\left(\bar{R}, \hat{x}^{1}\right)$.

\footnotetext{
${ }^{14}$ Some of the constructions used in this and the following two steps of the proof are illustrated in Figure 3.
} 
By Lemma $5, \operatorname{Pr}_{1} \mathcal{P}((\bar{R}, \hat{R}, \ldots, \hat{R}), \Omega)=\operatorname{Pr}_{1} \mathcal{P}_{2}((\bar{R}, \hat{R}), \Omega)$. If $\left(x^{1}, \Omega-\right.$ $\left.x^{1}\right) \in X_{2}^{*}$, Pareto optimality implies that $\nabla \hat{U}\left(\Omega-x^{1}\right)$ is proportional to $\nabla \bar{U}\left(x^{1}\right)$. From Step 5 we know that $\nabla \bar{U}\left(x^{1}\right) \in K_{p}$ for all $x^{1} \in \operatorname{Pr}_{1} X_{2}$. Thus, because $\nabla \hat{U}\left(\Omega-x^{1}\right) \notin K_{p}$ for any $\left(\Omega-x^{1}\right) \in \mathbb{R}_{+}^{m} \backslash K^{*}$ for which $\left(\Omega-x^{1}\right) \neq 0_{m}$, $\left(x^{1}, \Omega-x^{1}\right) \in \mathcal{P}_{2}((\bar{R}, \hat{R}), \Omega) \cap X_{2}^{*}$ implies that $x^{1} \in\left(\Omega-K^{*}\right) .{ }^{15}$ By Lemma 7, for all $x^{1} \in \operatorname{Pr}_{1}\left[\mathcal{P}_{2}((\bar{R}, \hat{R}), \Omega) \cap X_{2}^{*}\right], \operatorname{Pr}_{1} \mathcal{P}_{2}((\bar{R}, \hat{R}), \Omega)$ is increasing from person 1's perspective at $x^{1}$. Because preferences are classical, $\mathcal{P}_{2}((\bar{R}, \hat{R}), \Omega)$ is a connected set. ${ }^{16}$ Hence, because $\Omega-K^{*}$ is a convex cone with vertex at $\Omega$ containing $x^{* 1}, x^{1}$ must be in the closure of $\Omega-K^{*}$ if $x^{1} \in \operatorname{Pr}_{1} \mathcal{P}_{2}((\bar{R}, \hat{R}), \Omega)$, $x^{1} \gg x^{* 1}$, and $\left(x^{1}, \Omega-x^{1}\right) \in X_{2} \backslash X_{2}^{*}$. Because of how $K^{*}$ is constructed in Step 6, if $\left(x^{1}, \Omega-x^{1}\right) \in X_{2} \backslash X_{2}^{*}, x^{1}$ is in the closure of $\Omega-K^{*}$, and $x^{1} \gg x^{* 1}$, then $x^{1}=\Omega$. Because $x^{* 1} \gg 0_{m}$ and $x^{1} \gg x^{* 1}$ for all $x^{1} \in\left(\Omega-K^{*}\right) \cap$ $U C\left(\bar{R}, \hat{x}^{1}\right)$, it then follows that $U C\left(\bar{R}, \hat{x}^{1}\right) \cap \operatorname{Pr}_{1} \mathcal{P}_{2}((\bar{R}, \hat{R}), \Omega) \subseteq\left(\Omega-K^{*}\right) \cup \Omega$.

By minimum consumption guarantee, $\bar{x}^{1} \neq \Omega$. Because $\bar{x}^{1} \in U C\left(\bar{R}, \hat{x}^{1}\right) \cap$ $\operatorname{Pr}_{1} \mathcal{P}_{2}((\bar{R}, \hat{R}), \Omega)$, we have shown that $\bar{x}^{1} \in\left(\Omega-K^{*}\right) \cap U C\left(\bar{R}, \hat{x}^{1}\right)$.

Step 9. By Step 7 we know that $x^{1} \gg x^{* 1}$ for all $x^{1} \in\left(\Omega-K^{*}\right) \cap$ $U C\left(\bar{R}, \hat{x}^{1}\right)$. By Step $8, \bar{x}^{1} \in\left(\Omega-K^{*}\right) \cap U C\left(\bar{R}, \hat{x}^{1}\right)$. Hence, $\bar{x}^{1} \gg x^{* 1}$. But this inequality contradicts Lemma 1 because $\bar{x}^{1}=f^{1}(\bar{R}, \hat{R}, \ldots, \hat{R})$ and $x^{* 1}=f^{1}\left(R^{*}, \hat{R}, \ldots, \hat{R}\right)$. Therefore, $f$ cannot satisfy strategy-proofness, Pareto optimality, and minimum consumption guarantee on the domain $\left(\mathcal{R}^{C H S}\right)^{n}$.

(b) Now, suppose that $\mathcal{R}^{C H S} \subset \mathcal{D} \subseteq \mathcal{R}$. Contrary to the Theorem, suppose that $f$ satisfies strategy-proofness, Pareto optimality, and minimum consumption guarantee. Let $f^{*}$ denote the restriction of $f$ to the domain $\left(\mathcal{R}^{C H S}\right)^{n}$. The function $f^{*}$ also satisfies all three axioms, contradicting what was established in part (a) of the proof.

\section{Concluding Remarks}

We have shown that an efficient strategy-proof social choice function cannot guarantee minimum consumption in exchange economies with classical economic preferences. Thus, there is a fundamental conflict between efficiency and distributional goals if the collective choice procedure is required to provide incentives for individuals to always truthfully reveal their preferences.

\footnotetext{
${ }^{15}$ Because $\Omega \notin K^{*}, \hat{x}^{1}$ is proportional to $\Omega$, and $\left(\hat{x}^{1}, \Omega-\hat{x}^{1}\right) \in X_{2}^{*}$, it follows from this observation that $\overline{\mathbf{x}} \neq \hat{\mathbf{x}}$.

${ }^{16}$ See Mas-Colell, Whinston, and Green [10, p. 541].
} 
It would be socially undesirable to permit any individual to have a consumption bundle arbitrarily close to the origin. We therefore conclude that in order for a social choice function to be acceptable, departures from strategyproofness and/or Pareto optimality must be condoned. Barberà and Jackson [1] and Roberts and Postlewaite [14], among others, have made important contributions to our understanding of the tradeoffs that are possible between strategy-proofness and Pareto optimality in exchange economies, but much more remains to be learned.

\section{References}

1. S. Barberà and M. O. Jackson, Strategy-proof exchange, Econometrica 63 (1995), 51-87.

2. P. S. Dasgupta, P. J. Hammond, and E. S. Maskin, The implementation of social choice rules: Some general results on incentive compatibility, Rev. Econ. Stud. 46 (1979), 185-216.

3. L. Hurwicz, On informationally decentralized systems, in "Decision and Organization: A Volume in Honor of Jacob Marschak" (C. B. McGuire and R. Radner, Eds.), pp. 297-336, North-Holland, Amsterdam, 1972.

4. L. Hurwicz and M. Walker, On the generic nonoptimality of dominantstrategy allocation mechanisms: A general theorem that includes pure exchange economies, Econometrica 58 (1990), 683-704.

5. B.-G. Ju, Strategy-proofness versus efficiency in exchange economies: General domain properties and applications, unpublished manuscript, Department of Economics, University of Kansas, 2001.

6. B.-G. Ju, Continuous selections from the Pareto correspondence and non-manipulability in exchange economies, unpublished manuscript, Department of Economics, University of Kansas, 2001.

7. Y. Kannai, Continuity properties of the core of a market, Econometrica 38 (1970), 791-815.

8. M. Kato and S. Ohseto, Towards general impossibilty theorems in pure exchange economies, Soc. Choice Welfare 19 (2002), 659-664.

9. M. Kato and S. Ohseto, Non-dummy agents in pure exchange economies, unpublished manuscript, Faculty of Economics, Tokyo Metropolitan University, 2002.

10. A. Mas-Colell, M. D. Whinston, and J. R. Green, "Microeconomic Theory," Oxford Univ. Press, New York, 1995. 
11. E. Maskin, Nash equilibrium and welfare optimality, Rev. Econ. Stud. 66 (1999), 23-38.

12. H. Moulin and W. Thomson, Can everyone benefit from growth? Two difficulties, J. Math. Econ. 17 (1988), 339-345.

13. E. Muller and M. A. Satterthwaite, The equivalence of strong positive association and strategy-proofness, J. Econ. Theory 14 (1977), 412418.

14. D. J. Roberts and A. Postlewaite, The incentives for price-taking behavior in large exchange economies, Econometrica 44 (1976), 115-127.

15. M. A. Satterthwaite, Strategy-proofness and markets, Soc. Choice Welfare 18 (2001), 37-58.

16. M. A. Satterthwaite and H. Sonnenschein, Strategy-proof allocation mechanisms at differentiable points, Rev. Econ. Stud. 48 (1981), 587597.

17. J. Schummer, Strategy-proofness versus efficiency on restricted domains of exchange economies, Soc. Choice Welfare 14 (1997), 47-56.

18. S. Serizawa, Pairwise strategy-proofness, Discussion Paper No. 152, Tohoku Economics Research Group, Faculty of Economics, Tohoku University, 2000.

19. S. Serizawa, Inefficiency of strategy-proof rules for pure exchange economies, J. Econ. Theory, forthcoming.

20. Y. Sprumont, A note on strategyproofness in Edgeworth-box economies, Econ. Letters 49 (1997), 45-50.

21. L. Zhou, Inefficiency of strategy-proof allocation mechanisms in pure exchange economies, Soc. Choice Welfare 8 (1991), 247-254. 


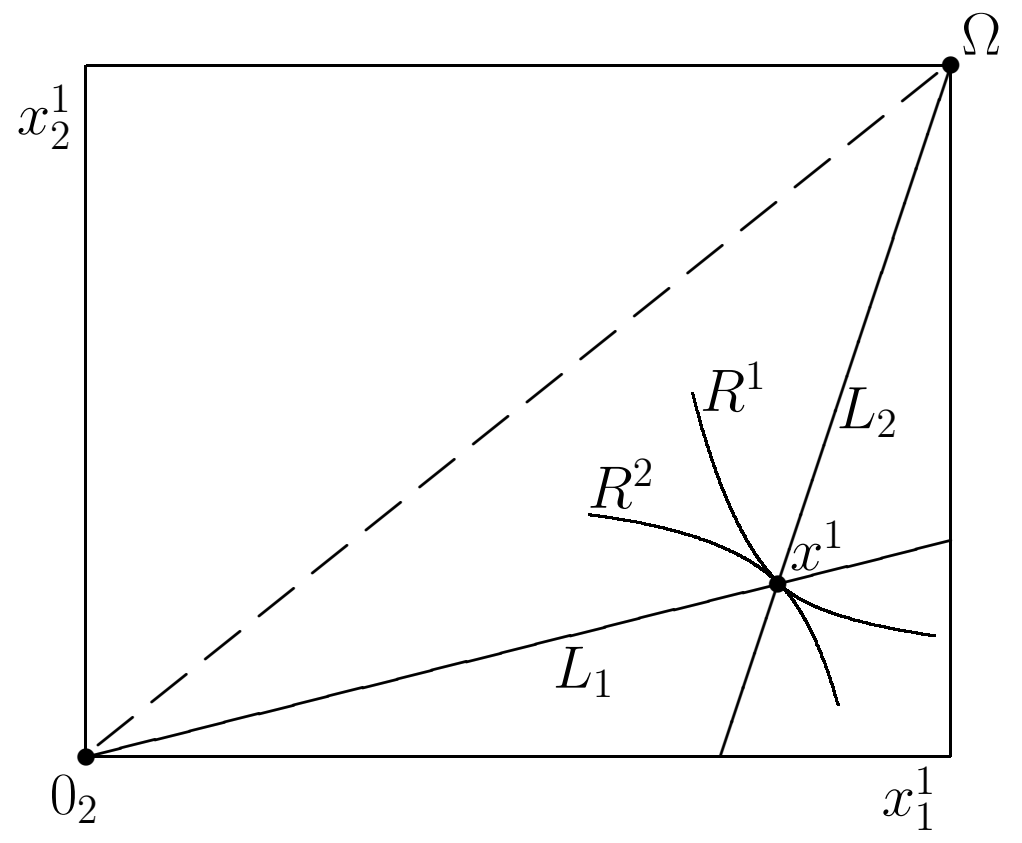

Figure 1 


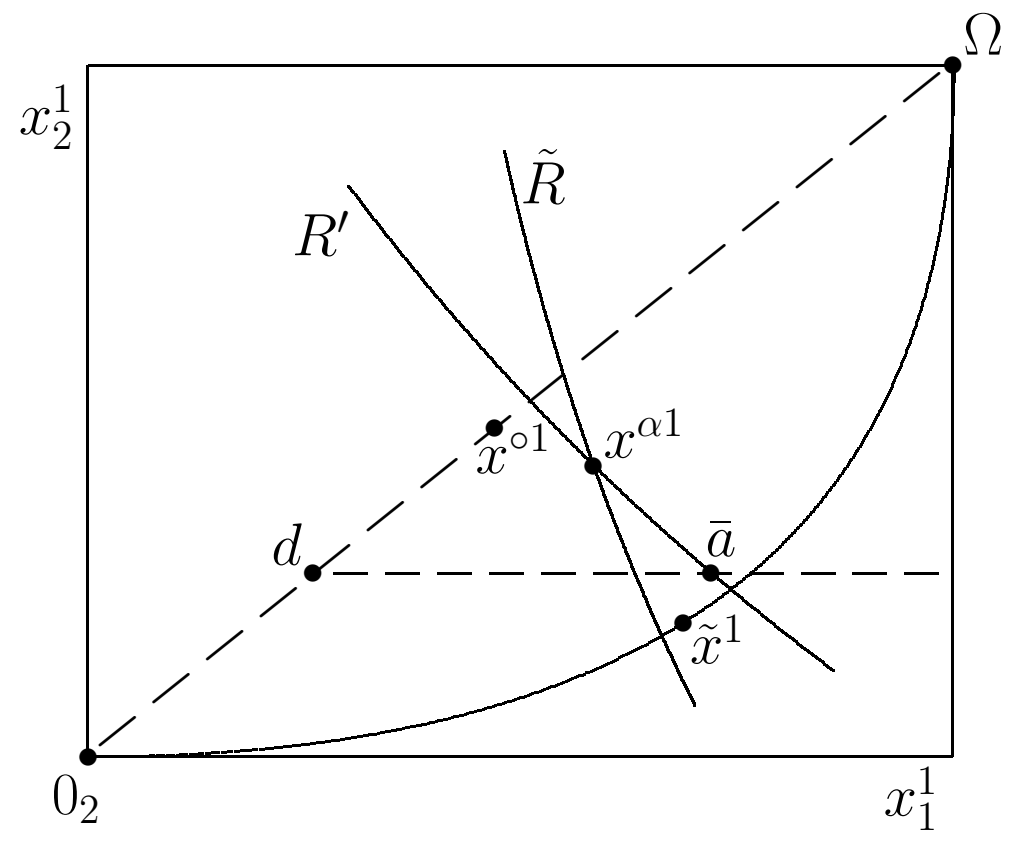

Figure 2 


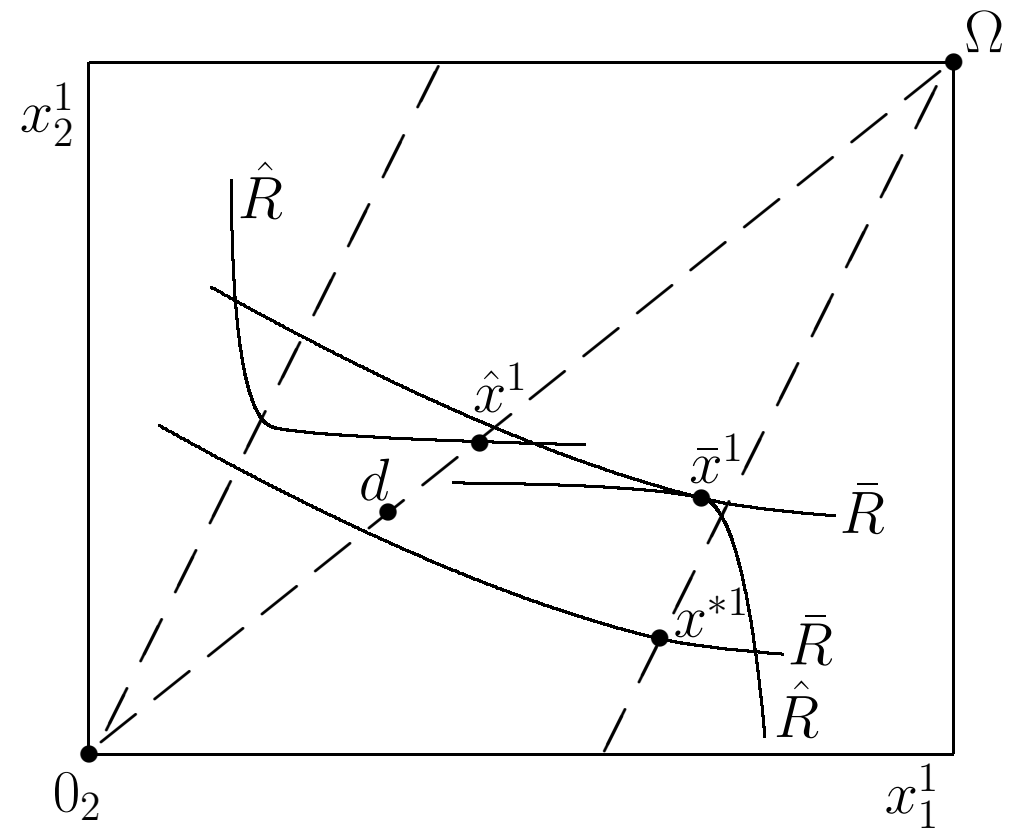

Figure 3 